\title{
Tailored electron bunches with smooth current profiles for enhanced transformer ratios in beam-driven acceleration
}

\author{
F. Lemery ${ }^{1}$ and P. Piot ${ }^{1,2}$ \\ ${ }^{1}$ Northern Illinois Center for Accelerator and Detector Development and Department of Physics, \\ Northern Illinois University, DeKalb, Illinois 60115, USA \\ ${ }^{2}$ Accelerator Physics Center, Fermi National Accelerator Laboratory, Batavia, Illinois 60510, USA
}

(Received 22 May 2015; published 3 August 2015)

\begin{abstract}
Collinear high-gradient $\mathcal{O}(\mathrm{GV} / \mathrm{m})$ beam-driven wakefield methods for charged-particle acceleration could be critical to the realization of compact, cost-efficient, accelerators, e.g., in support of TeV-scale lepton colliders or multiple-user free-electron laser facilities. To make these options viable, the high accelerating fields need to be complemented with large transformer ratios $>2$, a parameter characterizing the efficiency of the energy transfer between a wakefield-exciting "drive" bunch to an accelerated "witness" bunch. While several potential current distributions have been discussed, their practical realization appears challenging due to their often discontinuous nature. In this paper we propose several alternative continuously differentiable (smooth) current profiles which support enhanced transformer ratios. We especially demonstrate that one of the devised shapes can be implemented in a photo-emission electron source by properly shaping the photocathode-laser pulse. We finally discuss a possible superconducting linear-accelerator concept that could produce shaped drive bunches at high-repetition rates to drive a dielectric-wakefield accelerator with accelerating fields on the order of $\sim 60 \mathrm{MV} / \mathrm{m}$ and a transformer ratio $\sim 5$ consistent with a recently proposed multiuser free-electron laser facility.
\end{abstract}

DOI: 10.1103/PhysRevSTAB.18.081301

PACS numbers: 29.20.Ej, 29.27.-a, 41.85.-p, 41.75.Fr

\section{INTRODUCTION}

In beam-driven techniques, a high-charge "drive" bunch passes through a high-impedance medium and experiences a decelerating field [1-3]. The resulting energy loss can be transferred to a properly delayed "witness" bunch trailing the drive bunch. A critical parameter associated to this class of acceleration method is the transformer ratio

$$
\mathcal{R} \equiv\left|\frac{E_{+}}{E_{-}}\right|
$$

where $E_{+}$is the maximum accelerating field behind the drive bunch, and $E_{-}$is the maximum decelerating field within the drive bunch.

Generally, the transformer ratio is limited to values $\mathcal{R} \leq 2$ due to the fundamental beam-loading theorem [4]. However larger values can be produced using drive bunches with tailored (asymmetric) current profiles. Furthermore, it can be shown that both $\mathcal{R}$ and $E_{+}$for a given charge are maximized when the decelerating field over the drive bunch is constant [5]. Additionally, bunch current profiles that minimize the accumulated energy

Published by the American Physical Society under the terms of the Creative Commons Attribution 3.0 License. Further distribution of this work must maintain attribution to the author(s) and the published article's title, journal citation, and DOI. spread within the drive bunch are desirable as they enable transport of the drive bunch over longer distances.

To date, several current profiles capable of producing transformer ratios $\mathcal{R}>2$ have been theoretically explored. These include linearly-ramped current profiles combined with a door-step or exponential initial distribution [6]. An alternative method involves the use of a train of drive bunches (instead of a shaped single drive bunch) with appropriate charge and separation $[5,7,8]$. To date enhanced transformer ratios were experimentally demonstrated using the latter bunch-train method [8] and resulted in $\mathcal{R} \simeq 3.4$ [9]. Producing and transporting a train of bunches with different charges has its own sets of complications. Consequently, there has been regained interests in devising alternative techniques to shape the current distribution of drive bunches. Most recently a piecewise "double-triangle" current profile was suggested as an alternative to the linear-ramp distribution and its possible realization was numerically demonstrated [10] by implementing a transverse-to-longitudinal phase-space exchanger [11]. A limitation common to the formation of all the proposed shapes resides in their discontinuous character which makes their experimental realization either challenging or relying on complicated beam-manipulation techniques $[11,12]$. In addition these shapes are often foreseen to be formed in combination with an interceptive mask $[13,14]$ which add further challenges when combined with high-repetition-rate linacs [15].

In this paper we introduce several smooth current profiles which support large transformer ratios and lead 
to quasiconstant decelerating fields across the drive bunch. We describe a simple scheme for realizing one of these shapes in a photoemission radio frequency (rf) electron source employing a shaped photocathodelaser pulse. Finally, we discuss a possible injector configuration that could form drive bunches consistent with the multiuser free-electron laser (FEL) studied in Ref. [15].

\section{SMOOTH SHAPES}

For simplicity we consider a wakefield-assisting medium (e.g., a plasma or a dielectric-lined waveguide) that supports an axial wakefield described by the Green's function [16]

$$
G(\zeta)=2 \kappa \cos (k \zeta)
$$

where $\kappa \equiv \frac{|V|^{2}}{4 W}$ is the loss factor ( $V$ and $W$ are, respectively, the voltage and stored energy associated to the excited mode), $k \equiv 2 \pi / \lambda$ with $\lambda$ being the wavelength of the considered mode. Here $\zeta>0$ (in our convention) is the distance behind the source particle responsible for the wakefield. In this section we do not specialize to any wakefield mechanism and recognize that, depending on the assisting medium used to excite the wakefield, many modes might be excited so that the Green's function would consequently consist of a summation over these modes [and the various quantities appearing in Eq. (2) would have to be properly indexed and associated to the various excited modes].
Given the Green's function, the voltage along and behind a bunch with axial charge distribution $S(z)$ can be obtained from the convolution [16]

$$
V(z)=\int_{-\infty}^{z} G(z-\zeta) S(\zeta) d \zeta
$$

We take $S(z)$ to be nonvanishing on two intervals $[0, \xi]$ and $[\xi, Z]$ and zero elsewhere. In our convention the bunch head starts at $z=0$ and the tail population lies at $z>0$. We also constrain our search to functions such that $S(z)$ and $S^{\prime}(z) \equiv d S / d z$ are continuous at $z=\xi$. Introducing the function $f(z)$ (to be specified later), we write the charge distribution as

$$
S(z)= \begin{cases}f(z) & \text { if } 0 \leq z<\xi \\ f^{\prime}(\xi) z-f^{\prime}(\xi) \xi+f(\xi) & \text { if } \xi \leq z \leq Z \\ 0 & \text { elsewhere }\end{cases}
$$

\section{A. Linear ramp with sinusoidal head}

Based on our previous work [17] we first consider the following function

$$
f(z)=a z+b \sin (q k z),
$$

where $a$ and $b$ are positive constants, $k$ is again the spatial frequency seen above, and $q>0$ is an integer. Consequently, using Eq. (4), the axial bunch profile is written as

$$
S(z)= \begin{cases}a z+b \sin (q k z) & \text { if } 0 \leq z<\xi \\ a z+b q k(z-\xi) \cos (q \xi k)+b \sin (q \xi k) & \text { if } \xi \leq z \leq Z \\ 0 & \text { elsewhere }\end{cases}
$$

In this section we report only on solutions pertaining to $\xi=\lambda / 2$. Additional, albeit more complicated, solutions also exist for larger $\xi$; however, these solutions lead to additional oscillations which ultimately lowers the transformer ratio.

From Eq. (3), the decelerating field then takes the form

$$
E_{-}(z)=\kappa \begin{cases}\frac{\lambda}{\pi^{2}}\left(a \lambda \sin ^{2}\left(\frac{\pi z}{\lambda}\right)+\frac{\pi b q\left(\cos \left(\frac{2 \pi z}{\lambda}\right)-\cos \left(\frac{2 \pi q z}{\lambda}\right)\right)}{q^{2}-1}\right) & z<\lambda / 2 \\ \frac{\lambda\left(\left(q^{2}-1\right)\left(a \lambda+2 \pi b(-1)^{q} q\right)+\cos \left(\frac{2 \pi z}{\lambda}\right)\left(2 \pi b q\left((-1)^{q} q^{2}+1\right)-a\left(q^{2}-1\right) \lambda\right)\right)}{2 \pi^{2}\left(q^{2}-1\right)} & z \geq \lambda / 2\end{cases}
$$

The oscillatory part in the tail $(\lambda / 2 \leq z)$ can be eliminated under the condition

$$
b=\frac{a\left(q^{2}-1\right) \lambda}{2 \pi q\left((-1)^{q} q^{2}+1\right)}
$$

which leads to the following decelerating and accelerating fields, respectively 


$$
\begin{aligned}
E_{-}(z)=\kappa \begin{cases}\frac{a \lambda^{2}\left(2(-1)^{q} q^{2} \sin ^{2}\left(\frac{\pi z}{\lambda}\right)-\cos \left(\frac{2 \pi q z}{\lambda}\right)+1\right)}{2 \pi^{2}\left((-1)^{q} q^{2}+1\right)} & z<\lambda / 2 \\
\frac{a\left((-1)^{q}\left(2 q^{2}-1\right)+1\right) \lambda^{2}}{2 \pi^{2}\left((-1)^{q} q^{2}+1\right)} & z \geq \lambda / 2\end{cases} \\
E_{+}(z)=\int_{0}^{Z=N \lambda} G\left(z-z^{\prime}\right) S\left(z^{\prime}\right) d z^{\prime} \\
=\kappa \frac{a \lambda^{2}\left(\pi\left((-1)^{q}\left((4 N-1) q^{2}-2 N+1\right)+2 N\right) \sin \left(2 \pi\left(N-\frac{z}{\lambda}\right)\right)+\left((-1)^{q}\left(2 q^{2}-1\right)+1\right) \cos \left(2 \pi\left(N-\frac{z}{\lambda}\right)\right)\right)}{2 \pi^{2}\left((-1)^{q} q^{2}+1\right)},
\end{aligned}
$$

where, for convenience, we wrote the total bunch length as an integer number of the fundamental-mode wavelength $Z=N \lambda$. We note that $N$ does not have to be an integer number (it is generally a real-positive number) but this choice leads to simpler final equations and adheres to similar choice in the literature; see, e.g., [6,10]. Finally, the transformer ratio can be calculated by taking the ratio of the maximum accelerating field (see Appendix A) over the maximum decelerating field which yields

$$
\mathcal{R}=\frac{\sqrt{\pi^{2}\left((-1)^{q}\left((4 N-1) q^{2}-2 N+1\right)+2 N\right)^{2}+\left((-1)^{q}\left(2 q^{2}-1\right)+1\right)^{2}}}{(-1)^{q}\left(2 q^{2}-1\right)+1} .
$$

Two sets of solutions occur for even and odd $q$ which can be interpreted as a phase shift in the oscillatory part. Additionally, larger multiples of even and odd $q$ lead to more oscillations in the head which ultimately reduce the transformer ratio. In Fig. 1 we illustrate the simplest even (a) and odd (b) solutions corresponding to $q=2$ and $q=3$, respectively.

\section{B. Linear ramp with parabolic head}

We now consider an even simpler "quadratic" shape which was inspired by previous studies that seek to develop simple current shaping techniques based on the introduction of nonlinear correlations in the longitudinal phase

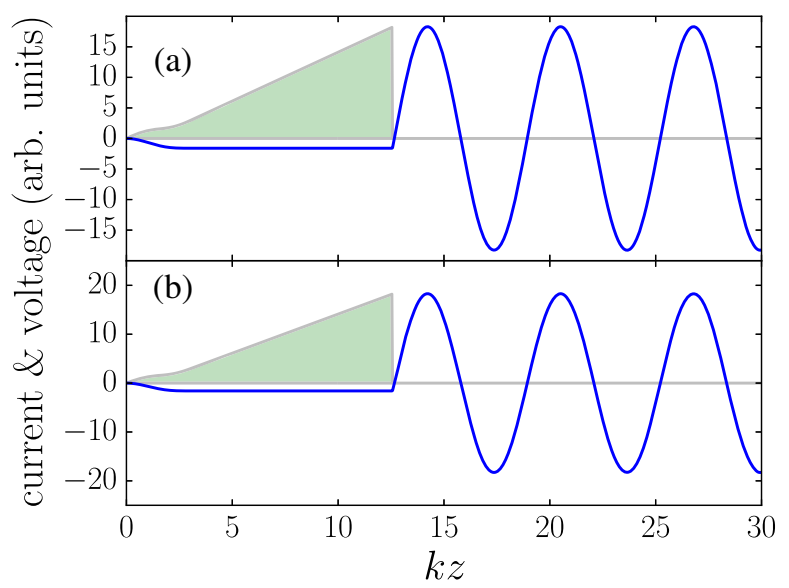

FIG. 1. Example of current profiles described by Eq. (6) (shaded line) with the corresponding induced voltages. The total bunch length is set to $Z=5 \lambda$ (i.e., $N=5$ ) and plots (a) and (b), respectively, correspond to the cases $q=2$ and $q=3$. The head of the bunch is at $k z=0$. space using a wakfield mechanism $[17,18]$. We especially consider the profile function

$$
f(z)=a z^{2},
$$

which yields the current profile

$$
S(z)= \begin{cases}a z^{2} & \text { if } 0 \leq z<\xi \\ 2 a \xi z-a \xi^{2} & \text { if } \xi \leq z \leq Z \\ 0 & \text { elsewhere }\end{cases}
$$

The resulting decelerating field within the bunch is

$$
E_{-}(z)=2 \kappa \begin{cases}-2 a \frac{\sin (k z)-k z}{k^{3}} & \text { if } 0 \leq z<\xi, \\ \frac{2 a}{k^{3}}\{\sin [k(z-\xi)]-\sin (k z)+2 k \xi\} & \text { if } \xi \leq z \leq Z, \\ 0 & \text { elsewhere. }\end{cases}
$$

Again, the decelerating field can be made constant for $z \in$ $[\xi, Z]$ when $\xi=\nu \lambda$ with $\nu \in \mathbb{N}$. In such a case the previous equation simplifies to

$$
E_{-}(z)=2 \kappa \begin{cases}-2 a \frac{\sin (k z)-k z}{k^{3}} & \text { if } 0 \leq z<\nu \lambda \\ \frac{4 \pi a \nu}{k^{3}} & \text { if } \nu \lambda \leq z \leq Z \\ 0 & \text { elsewhere. }\end{cases}
$$

The accelerating field trailing the bunch $(z>Z \equiv N \lambda)$ is

$$
E_{+}(z)=-\frac{8 \pi \nu a \kappa}{k^{3}}[\pi(2 N-\nu) \sin (k z)-\cos (k z)],
$$




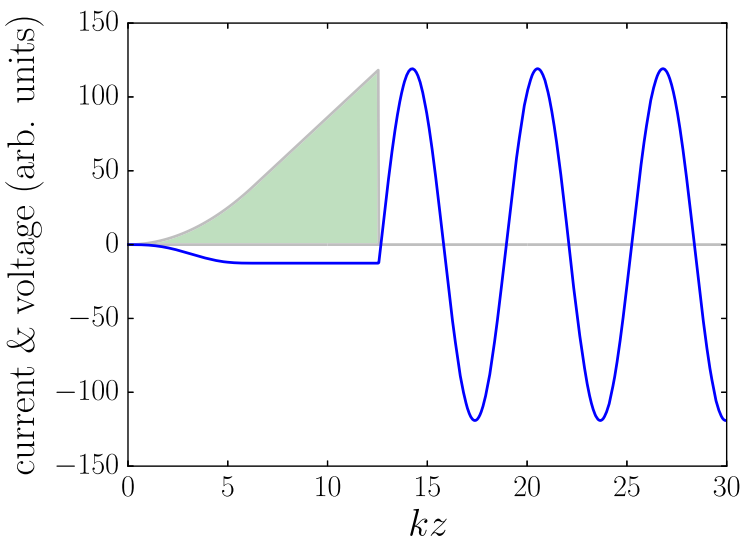

FIG. 2. Example of "quadratic" current profiles given by Eq. (13) (shaded line) with corresponding induced voltage. The parameters are $\nu=1$ and $N=5$. The head of the bunch is at $k z=0$.

yielding the transformer ratio

$$
\mathcal{R}=\left[1+\pi^{2}(2 N-\nu)^{2}\right]^{1 / 2} .
$$

In Fig. 2 we illustrate an example of the quadratic shape (green trace) as well as its corresponding longitudinal electric field (blue trace) for $\nu=1$ and $N=5$.

\section{Comparison with other shapes}

We now turn to compare the smooth longitudinal shapes from the previous section with the doorstep [6] and doubletriangle [10] which also provide constant decelerating fields over the bunch-tail (see Appendix B for our formulation of these distributions). For a fair comparison, we stress the importance of comparing the various current profiles with equal charge. Consequently, we normalize each of the current profile to the same bunch charge

$$
Q=\int_{0}^{Z=N \lambda} d z S(z, a)
$$

where $a$ is the scaling parameter associated with each bunch shape (see Sec. II and Appendix B), and $N \lambda$ is the

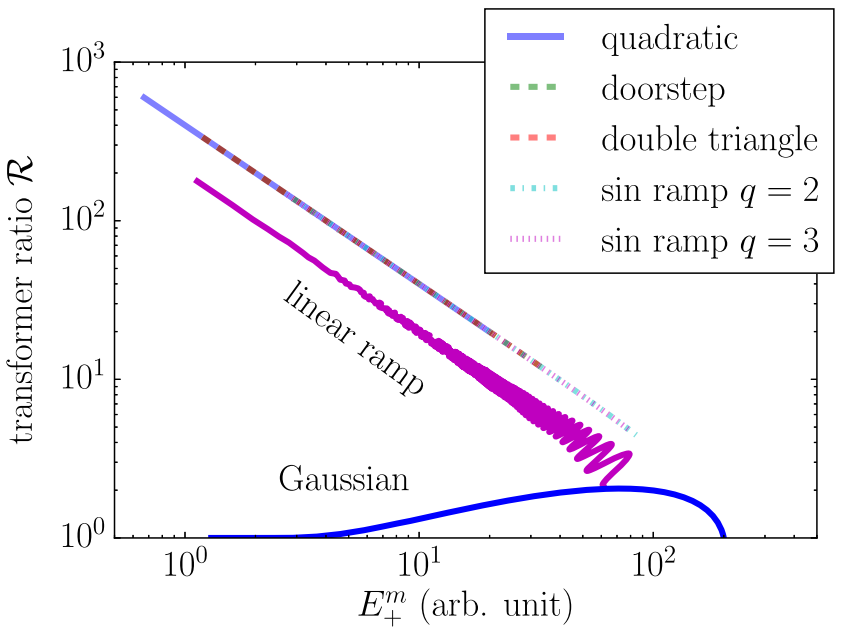

FIG. 3. Tradeoff curves between $\mathcal{R}$ and $E_{+}^{m}$ for the current profiles listed in Table I. The "quadratic" and "sin ramps" respectively correspond to the distributions proposed in Secs. II B and II A. The Gaussian and ramp distributions are displayed for comparison.

total bunch length which is assumed to be larger than the given shape's bunch-head length $(N \lambda>\xi)$. For each distribution, the charge normalization generates a relationship between $a$ and $N \lambda$ which enables us to reexpress $\mathcal{R}$ in terms of $Q$ and $a$. In Table I we tabulate the analytical results for $\mathcal{R}(N)$ (following the conventional notation $[6,10]$ ) and $\mathcal{R}(Q, a)$, and also list the maximum decelerating field $E_{-}^{m}$ for each distribution. Additionally in Fig. 3 we illustrate these results in a log-log plot where, for each distribution, the scaling parameter $(a)$ was varied for a fixed charge and wavelength. To complete our comparison we also added the linear-ramp and Gaussian distributions.

The results indicate that all of the distributions with constant decelerating fields over the bunch-tail have similar performances (i.e., they are all located on the same tradeoff curve in Fig. 3). Additionally, by varying the scaling parameter $a$ for a given distribution, one can shift the performance of the distribution to have a larger $\mathcal{R}$ at the expense of a small $E_{+}$and vice versa. Ultimately, this suggests that the distribution, which is simplest to produce

TABLE I. Table comparing several different proposed drive bunch distributions as a function of bunch length and charge. Additionally, the maximum decelerating field $\left(E_{-}^{m}\right)$ is shown for each distribution. Here we consider $\kappa=1$.

\begin{tabular}{lccr}
\hline \hline Distribution & $\mathcal{R}(N)$ & $\mathcal{R}(Q)$ & $E_{-}^{m}$ \\
\hline Doorstep [6] & $\sqrt{1+(1-\pi / 2+2 \pi N)^{2}}$ & $\sqrt{2+\pi\left(\frac{4 Q}{a \lambda}-1\right)}$ & $\frac{a \lambda}{\pi}$ \\
Double triangle [10] & $\sqrt{1+(2 \pi N-1)^{2}}$ & $\sqrt{2+\pi\left(\frac{4 Q}{a \lambda}-1\right)}$ & $\frac{a \lambda}{\pi}$ \\
Sin $(q=2)$ & $\frac{1}{8} \sqrt{\pi^{2}(3-16 N)^{2}+64}$ & $\frac{1}{8} \sqrt{64-15 \pi^{2}+\frac{48 \pi Q}{a \lambda}}$ & $\frac{16 a \lambda}{3 \pi}$ \\
Sin $(q=3)$ & $\frac{1}{2} \sqrt{\pi^{2}(1-4 N)^{2}+4}$ & $\frac{1}{6} \sqrt{44-9 \pi^{2}+\frac{24 \pi Q}{a \lambda}}$ & $\frac{6 a \lambda}{\pi}$ \\
Quadratic & $\sqrt{1+\pi^{2}(2 N-1)^{2}}$ & $\sqrt{1+\pi^{2}\left(\frac{4 Q}{a \lambda^{3}}-\frac{1}{3}\right)}$ & $\frac{a \lambda^{3}}{\pi^{2}}$ \\
\hline \hline
\end{tabular}


is as useful as any other and can be scaled accordingly to yield the desired $\left(\mathcal{R}, E_{+}\right)$couple, e.g., for a specific application. These results also confirm our previous numerical investigation of the tradeoffs between $\mathcal{R}$ and $E_{+}$for different current distributions [19].

\section{PHOTOEMISSION OF OPTIMAL SHAPES VIA LASER-SHAPING}

In this section we investigate the realization of the quadratic distribution discussed in Sec. II by longitudinally tailoring a laser pulse impinging on a photocathode in a photoinjector [20,21]. The resulting electron distribution is then accelerated in a rf-gun and expands via space charge forces. If the charge density of the emanating electron bunch is sufficiently low, the resulting distribution will be relativistically preserved through a drift; however for larger charge densities, the original longitudinal distribution will morph according to the integrated space charge forces inside the bunch. The setup we consider throughout this section is depicted in Fig. 4 and consists of a typical $1+\frac{1}{2}-$ cell BNL/SLAC/UCLA S-band rf-gun operating at $2.856 \mathrm{GHz}$ surrounded by a solenoidal lens [22]. The large $(\sim 140 \mathrm{MV} / \mathrm{m})$ acceleration gradients in the gun help preserve larger charge densities compared with, e.g., L-band guns. The simulations are carried with ASTRA [23], a particle-in-cell beam-dynamics program that includes a quasistatic cylindrically-symmetric space charge algorithm. The simulation also includes the image-charge effect which arises during the photoemission process, in our simulations the electron bunch is represented by 200,000 macroparticles.

\section{A. Case of an ideal laser-shaping technique}

We base our approach on Ref. [24] where we developed a simple one-dimensional longitudinal space charge model to investigate the expansion forces in various distributions.

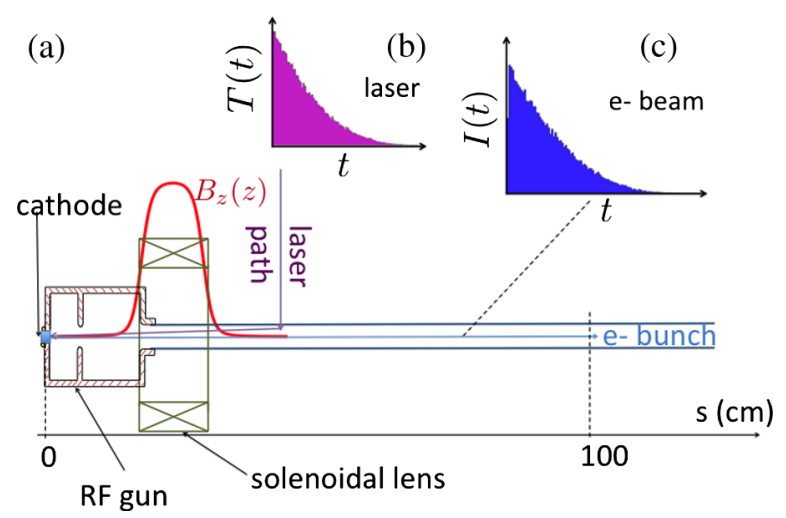

FIG. 4. Configuration used for the pulse-shaping simulations using a S-band rf gun (a). A temporally shaped laser pulse (b) is optimized to result in a photo-emitted electron-beam with current profile (c) having features similar to the distribution discussed in Sec. II B. In insets (a) and (b) the tail of the bunch is at $t=0$.
For power distributions of the form $z^{\alpha}$ where $\alpha$ is a realpositive number, we observed that the relatively small fields in the bunch-head $(z \sim 0)$ will essentially preserve the local longitudinal form. In the tail of the bunch however, there is an asymmetrical blowout which has proper sign to possibly lead to a linear-like tail which is required for the quadratic ramp.

We consider a laser intensity distribution of the form $I(r, t)=T(t) R(r)$, where $T(t)$ is the temporal profile and $R(r)$ the transverse laser envelope. In our previous studies we used a radially uniform transverse distribution and explored polynomial and exponential forms for $T(t)$. In this section we report on the performance of the polynomial distribution given by

$$
T(t)=T_{0} t^{\alpha} H(\tau-t)
$$

where $T_{0}$ is a normalization constant, $\alpha>0$ is the polynomial power, $\tau$ is the ending time of the pulse, and $H(t)$ is the Heaviside function.

The exponent $\alpha$ greatly influences the space-charge fields. Large values of $\alpha$ (e.g $\alpha \gtrsim 5$ ) lead to large spacecharge forces which results in a uniformly filled ellipsoidal distribution [25]. Alternatively, smaller values of $\alpha(\alpha \lesssim 3)$ lead to a more uniform evolution of the bunch dynamics due to the increased uniformity of the field over the bunch. Additionally, the transverse spot size of the laser pulse on the photocathode also controls the longitudinal electric fields but also influences the transverse "thermal" emittances. It is also possible to reduce the electric fields and the associated blowout rate by using longer laser pulses; in this scenario, the resulting electron bunch will evolve at a slower rate but the resulting bunch distribution will have a smaller peak current compared to when starting with smaller values of $\tau$. A smaller current will impact the performances of the wakefield accelerator (or require the implementation of a longitudinal compression scheme). Finally, it would also be possible to use a longer, e.g., $2+\frac{1}{2}-$ cell, rf gun or another acceleration cavity in close proximity to the gun to preserve larger charge densities which could effectively alleviate the need for a bunch compressor to drive large accelerating fields in the subsequent wakefield accelerator.

Figure 5 shows simulated longitudinal phase space snapshots and corresponding currents at different axial locations downstream of the gun for a 1-nC bunch. For this simulation a 1-mm rms laser spot size on the photocathode was used. The initial laser distribution was described by Eq. (19) with $\alpha=2$ and $\tau=15$ ps. A fit of the current distribution at $s=50 \mathrm{~cm}$ from the photocathode is shown in Fig. 5 and indicates that the final electron bunch distribution is indeed accurately described by Eq. (13). 

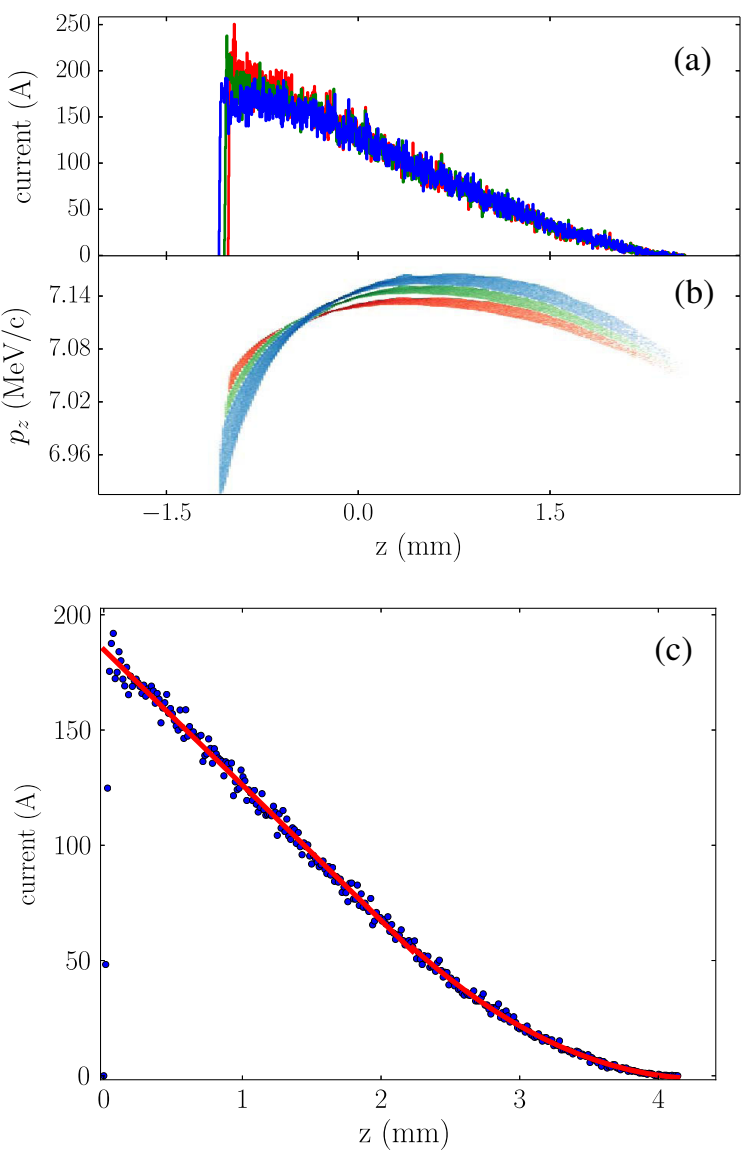

FIG. 5. Evolution of the electron-bunch current (a) and longitudinal phase space (b) along the beam line at 20 (red), 60 (green), and $100 \mathrm{~cm}$ (blue) from the photocathode surface and (c) comparison of the current profile numerically simulated at $s=50 \mathrm{~cm}$ (blue symbols) with a fit to Eq. (13) (red line). The head of the bunch is at large values of $z$.

\section{B. Limitation of a practical laser shaping technique}

As a first step toward a realistic model for the achievable shaped we consider the photoemission process to be resulting from frequency tripling of a $\lambda_{0}=800-\mathrm{nm}$ amplifier infrared (IR) pulse impinging a fast-response time cathode (with typical work functions corresponding to ultraviolet photon energy $\sim \lambda_{0} / 3$ ). Such a setup is commonly used in rf photoinjectors such as the one discussed in the previous sections. We further assume that the frequency up-conversion process does not affect the original laser's temporal shape (e.g., the UV-pulse temporal shape is identical to the IR-pulse temporal shape). Under such an assumption, the formation of the "ideal" quadratic temporal shape discussed in the previous section is limited by the finite laser bandwidth and frequency response of the shaping process.

We consider an incoming amplified IR pulse with intensity $I_{\text {in }}(r, t)=I_{0}(r) \operatorname{sech}^{2}(t / \tau)$ downstream of the last-stage amplification, where $\tau$ is the laser pulse duration. We model the IR pulse laser-shaping process via the convolution $I_{\text {out }}(r, t)=\int_{-\infty}^{+\infty} I_{\text {in }}\left(r, t-t^{\prime}\right) R\left(t^{\prime}\right) d t^{\prime} \quad$ where $I_{\text {out }}(t)$ and $R(t)$ represent the shaped-pulse intensity and response function of the shaping method, respectively.

Given the desired output shape and incoming laser pulse profile, the response function of the shaping process has to be set to satisfy [26]

$$
\tilde{R}(\omega)=\frac{\tilde{I}_{\mathrm{out}}(\omega)}{\tilde{I}_{\mathrm{in}}(\omega)},
$$

where the upper tilde represents the Fourier transformation $\tilde{f}(\omega)=\int_{-\infty}^{+\infty} f(t) e^{i \omega t}$. In practice $I_{\text {in }}(\omega)$ is defined over a finite range of frequency $\omega=\omega_{0} \pm \frac{\delta \omega}{2}$ where $\omega_{0} \equiv$ $\frac{2 \pi c}{\lambda_{0}}$ is the central laser frequency and $\delta \omega \equiv \frac{\omega_{0}}{\lambda_{0}} \delta \lambda$ is the laser pulse bandwidth ( $\delta \lambda$ is the wavelength span of the pulse spectrum).

The typical shape considered in the previous section after laser shaping is shown in Fig. 6; the limited bandwidth has very little effect except for the well-known ringing effect at the sharp discontinuities [27]; see Fig. 6(b) and (c). Another potential limitation to our shaping scheme arises with a high-efficiency (semiconductor) photocathode. We consider as an example the case of $\mathrm{Cs}_{2} \mathrm{Te}$ photocathodes because of their wide use in high-current photoinjectors. The response-time limitation is investigated using the parametrized impulsional time response of $\mathrm{Cs}_{2} \mathrm{Te}$ described in Ref. [28] based on numerical simulations presented in Ref. [29]. The impulsional response is convolved with the distribution used in the previous section and the results are gathered in Fig. 6. Again this effect appears to be marginal.

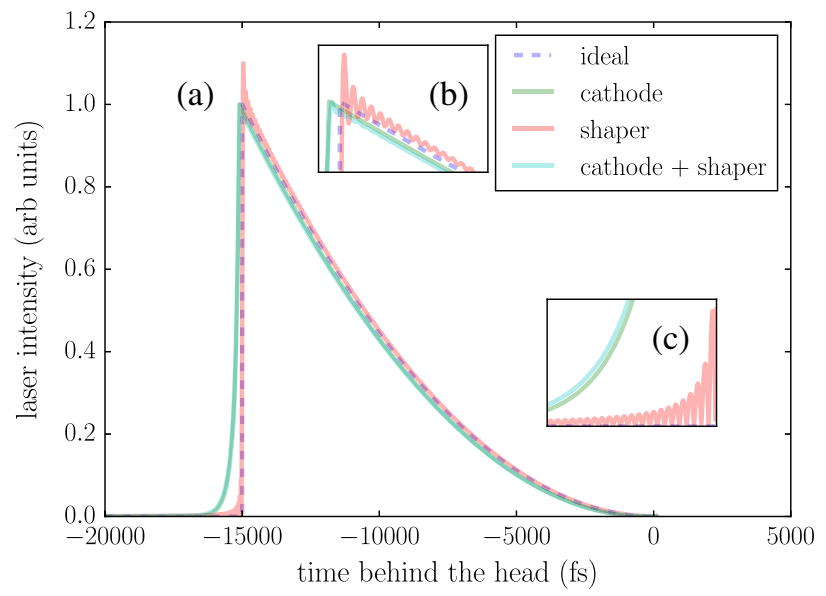

FIG. 6. Comparison of nominal ("ideal") quadratic shape with the shapes achieved when taking into account the photoemission response time ("cathode"), the laser-pulse-shaping finite bandwidth ("shaping"), and both effects ("cathode+shaper"). The ideal laser temporal profile is described by Eq. (19) with $\alpha=2$ and $\tau=15$ ps. Insets (b) and (c) are zooms of the areas $t \in$ $[-15200,-13600]$ fs (peak location) and $t \in[-16000,-15020] \mathrm{fs}$ (left edge of the profile), respectively. The head of the laser pulse is at $t=0$. 


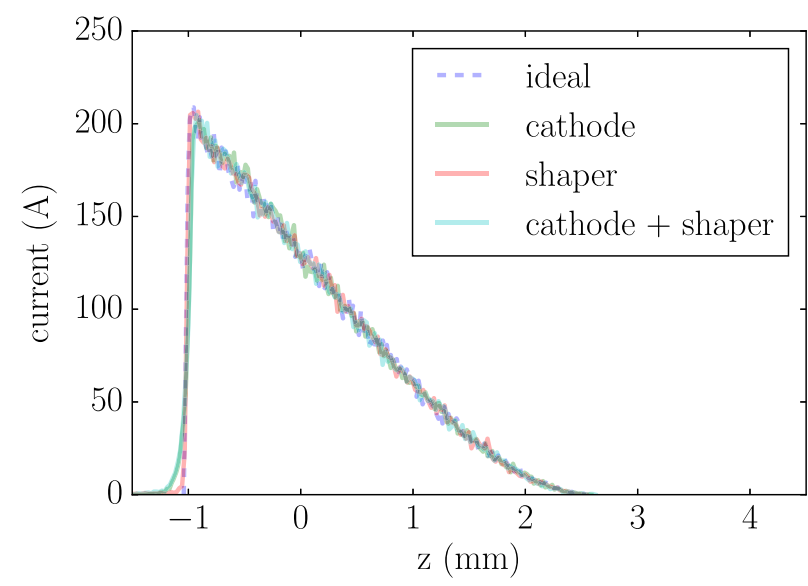

FIG. 7. Comparison of the final electron-bunch current at $s=$ $50 \mathrm{~cm}$ from the cathode surface for the four cases considered in Fig. 6. The "cathode" and "shaper" respectively correspond to the inclusion of the cathode response time and shaper bandwidth limitation in the initial particle distribution at $s=0$ while the ideal case is given by Eq. (19) with $\alpha=2$ and $\tau=15$ ps. The head of the bunch corresponds to $z>0$.

For the sake of completeness, the various profiles shown in Fig. 6 are tracked with ASTRA and the final current distributions at $s=50 \mathrm{~cm}$ are found to be indiscernibly close to the ideal shape considered in the previous section; see Fig. 7. Such a result gives further confidence in the proposed shaping approach.

\section{FORMATION OF HIGH-ENERGY TAILORED BUNCHES FOR A DWFA LINAC}

We finally investigate the combination of the tailored current-profile generation scheme with subsequent acceleration in a linac located downstream of the rf gun. Such a configuration could be useful to form tailored relativistic electron bunches for direct injection in wakefieldacceleration structures. For this example, we consider a high-repetition drive bunch with parameters consistent with a recently proposed beam-driven accelerator for a shortwavelength free-electron laser (FEL) [15]. We adopt a different approach than Ref. [15] and instead choose a 1.3-GHz superconducting RF (SCRF) linac (L0 and L1) composed of TESLA cavities [30] coupled to a quarterwave 200-MHz SCRF gun [31,32] originally designed for the WiFEL project [33]; see diagram in Fig. 8. The accelerator also includes a 3.9-GHz accelerating cavity (L39) section to remove nonlinearities in the longitudinal phase space $[34,35]$. For this study we explored the use of polynomial laser profile described by Eq. (19) and let $\alpha$ and $\tau$ as free parameters.

The laser-profile parameters and accelerator settings were optimized using a multiobjective genetic optimizer, GENETICOPTIMIZER available from Argonne National Laboratory [36], to seek a final distribution with current profile that achieves a high transformer ratio and relatively

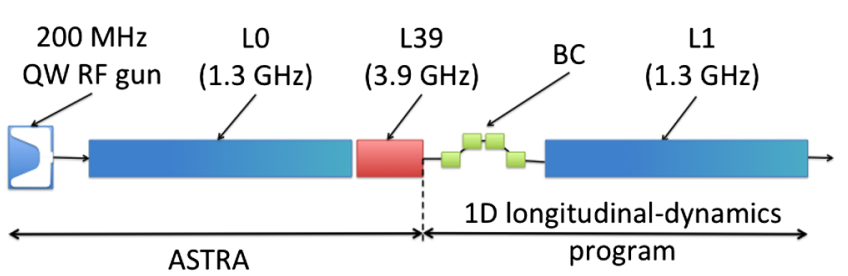

FIG. 8. Block diagram of the accelerator configuration explored for the formation of high-energy ramped bunches. The legend is as follows: "QW" stands for quarter-wave, "L0" and "L1" are standard $1.3-\mathrm{GHz}$ cryomodule equipped with 8 TESLA-type SCRF cavities, "L39" is a cryomodule consisting of four 3.9-GHz cavities, and "BC" is a magnetic bunch compressor.

large accelerating field. The program GENETICOPTIMIZER implements an evolutionary Pareto algorithm similar to the one described in Ref. [37]. The optimized accelerator settings are summarized in Table II. In our optimization, we chose the wakefield structure to be a dielectric-lined waveguide with parameters tabulated in Table III. The chosen relative electric permittivity $\epsilon_{r}=5.7$ corresponds to diamond. We constrain our optimization to ensure the entire beam is transmitted through the dielectric waveguide (the finite aperture of the waveguide is included in our simulations). Finally, we introduce a longitudinal scaling factor $\eta$ as a free parameter to model bunch compression, such that the axial coordinate is scaled following $z \rightarrow z^{\prime}=\eta z$. The optimization converged to a value $\eta=0.16$. The obtained wakefield and scaled shape are shown in Fig. 9(a). For the wakefield calculations we followed the formalism detailed in Ref. [38] and use the first four modes in the wake potential used for the beam dynamics simulations.

Given the devised configuration, a one-dimensional model of the longitudinal beam dynamics was employed to assess the viability of the required compression and especially explore the possible impact of nonlinearities in

TABLE II. Optimized settings for the accelerator parameters needed to produce and accelerate a drive bunch to $\sim 200 \mathrm{MeV}$. The parameter $\alpha$ and $\tau$ are defined in Eq. (19).

\begin{tabular}{lcc}
\hline \hline Parameter & Value & Units \\
\hline Laser rms spot size $\sigma_{r}$ & 2.5 & $\mathrm{~mm}$ \\
Laser ramp $\alpha$ parameter & 19.86 & - \\
Laser ramp duration $\tau$ & 96.8 & $\mathrm{ps}$ \\
Bunch charge $Q$ & 5 & $\mathrm{nC}$ \\
Peak E-field on cathode & 40 & $\mathrm{MV} / \mathrm{m}$ \\
Laser injection phase & $71.0 \mathrm{deg}$ & $(200 \mathrm{MHz})$ \\
Gun output beam momentum & 5.15 & $\mathrm{MeV} / \mathrm{c}$ \\
Accelerating voltage L0 & 165 & $\mathrm{MV} / \mathrm{m}$ \\
Off-crest phase L0 & $-12.35 \mathrm{deg}$ & $(1.3 \mathrm{GHz})$ \\
Accelerating voltage L39 & 24.1 & $\mathrm{MV}$ \\
Off-crest phase L39 & $-192.35 \mathrm{deg}$ & $(3.9 \mathrm{GHz})$ \\
Beam momentum after L39 & $\sim 143$ & $\mathrm{MeV} / \mathrm{c}$ \\
Final beam momentum after L1 & $\sim 350$ & $\mathrm{MeV} / \mathrm{c}$ \\
\hline \hline
\end{tabular}


TABLE III. Dielectric-line waveguide (DLW) parameters and resulting wakefield values using the current profile shown in Fig 9. The "ideal-" and "realistic-compression" entries respectively correspond to the cases when the final current profile is obtained via a simple longitudinal-axis scaling or via particle tracking.

\begin{tabular}{lcc}
\hline \hline Parameter, symbol & Value & Units \\
\hline DLW inner radius, $r_{i}$ & 750 & $\mu \mathrm{m}$ \\
DLW outer radius, $r_{o}$ & 795 & $\mu \mathrm{m}$ \\
DLW relative permittivity, $\epsilon_{r}$ & 5.7 & - \\
DLW fundamental mode, $f_{1}$ & 369.3 & $\mathrm{GHz}$ \\
Ideal compression: & & \\
Peak decelerating field, $\left|E_{-}\right|$ & 14.01 & $\mathrm{MV} / \mathrm{m}$ \\
Peak accelerating field, $\left|E_{+}\right|$ & 75.55 & $\mathrm{MV} / \mathrm{m}$ \\
Transformer ratio, $\mathcal{R}$ & 5.39 & \\
Realistic compression: & & \\
Peak decelerating field, $\left|E_{-}\right|$ & 12.84 & $\mathrm{MV} / \mathrm{m}$ \\
Peak accelerating field, $\left|E_{+}\right|$ & 63.87 & $\mathrm{MV} / \mathrm{m}$ \\
Transformer ratio, $\mathcal{R}$ & 4.95 & \\
\hline \hline
\end{tabular}

the longitudinal phase space on the achieved current profile. We considered the current could be longitudinally compressed using a conventional magnetic bunch compressor (BC) with longitudinal linear and second order dispersions $R_{56}$ and $T_{566} \equiv-\frac{3}{2} R_{56}$ [39]. In our simulations the longitudinal dispersion was taken to $R_{56}=-20 \mathrm{~cm}$ following similar designs [40]. The phase of L0 and phase and amplitude of L39 were empirically optimized and the resulting longitudinal phase space $\left(z_{0}, \delta_{0}\right)$ was tracked through the $\mathrm{BC}$ via the transformation $z_{0} \rightarrow z=$ $z_{0}+R_{56} \delta_{0}+T_{566} \delta_{0}^{2}$. An optimum set of phases and amplitudes was found and listed in Table II and the sequence of the longitudinal phase spaces along the injector appear in Fig. 10. The final wakefield excited in the structure with parameters listed in Table III is displayed in Fig. 9(b) - the achieved field and transformer ratio

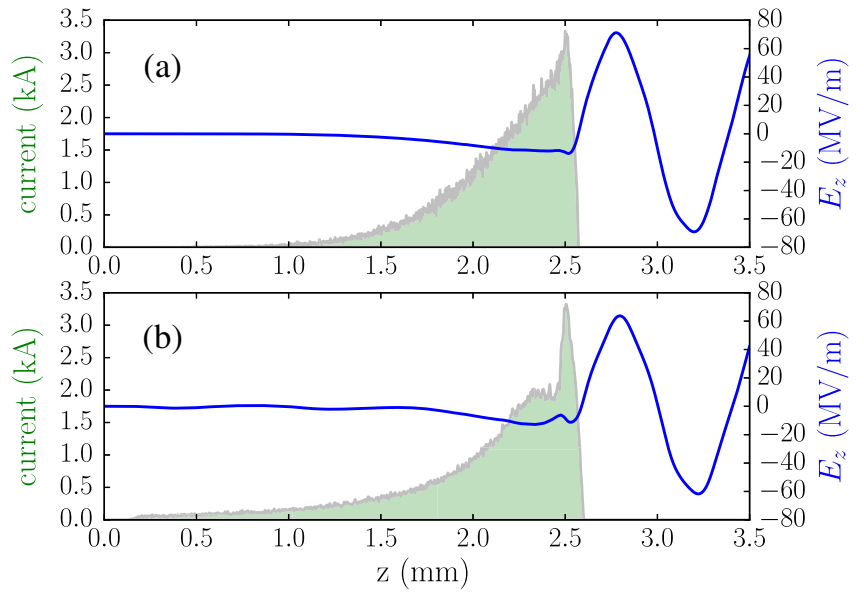

FIG. 9. Final current distribution (green shaded area) and associated wakefield (blue traces) for the "ideal" (a) and "realistic" (b) cases of compression discussed in the text. The head of the bunch corresponds to $z=0$.
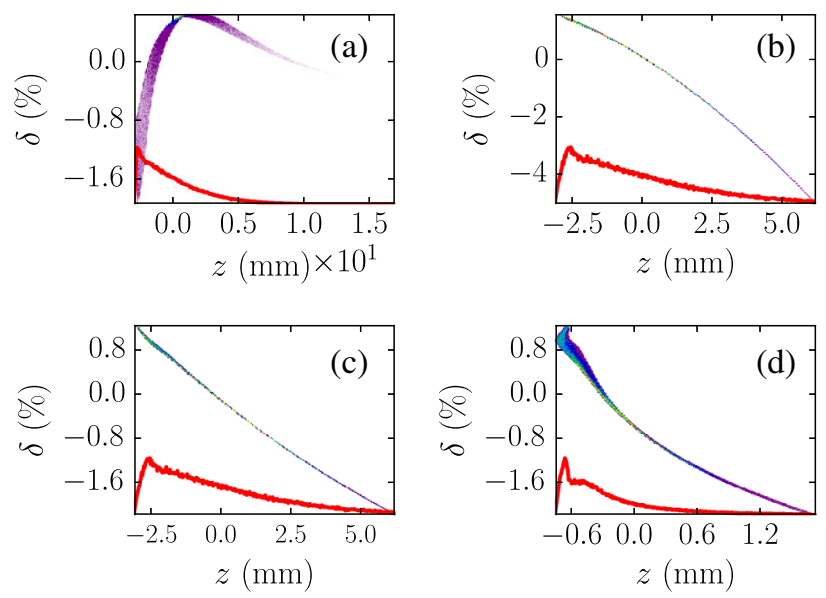

FIG. 10. Snapshots of the longitudinal phase spaces and associate current profiles (red traces) upstream of L0 (a) and downstream of L0 (b), L39 (c) and BC (d). Simulations up to L39 are carried with ASTRA whereas a one-dimensional longitudinaldynamics model is used for BC2. The head of the bunch corresponds to $z>0$.

values are summarized in Table III. We remark that the inclusion of a refined model of longitudinal dynamics leads to the apparition of features [e.g., a small current spike in the bunch tail; see Fig. 9(b) or 10(d)] that were absent in the optimization process implementing a simple scaling of the longitudinal coordinates; see Fig. 9(a). The origin of the small current spike can be traced back to the nonlinear correlation imposed by space charge in the early stages of the bunch-transport process (i.e., in the drift space upstream of L0); see Fig. 10(a). Nevertheless the achieved peak field and transformer ratio as the bunch passes through the DLW are very close (within 10\%) to the ones obtained with the scaled distribution. These results indicate that our proposed injector concept appears to produce the required current profile. Further studies, including a transverse beam dynamics optimization and the inclusion of collective effects such as coherent synchrotron radiation and space charge downstream of L39 and throughout the bunch compressor, will be needed to formulate a detailed design of the injector. We nevertheless stress that the simple model presented above confirms a plausible longitudinal-beam-dynamics capable of preserving the formed current profiles after acceleration and compression. The final energies and peak currents are all within the parameters suggested in Ref. [15].

We finally note that the generated current profiles are capable of supporting electric fields and transformer-ratios in a DLW structure with performances that strike a balance between the two cases listed as "case 1" and "case 2" in Table 1 of Ref. [15]; see Table III.

\section{SUMMARY}

In conclusion, we have presented a set of smooth current profiles for beam-driven acceleration which displays 
comparable performances with more complex discontinuous shapes discussed in previous work. We find that all proposed current profiles which lead to uniform decelerating fields are on the same $\left(E_{+}, \mathcal{R}\right)$ performance curve and that a given profile can be scaled to a particular accelerating field or transformer ratio. We also presented a simple lasershaping technique combined with a photoinjector to generate our proposed quadratic current profile. We finally illustrated the possible use of this technique to form an electron bunch with a tailored current profile. The distribution obtained from these start-to-end simulations was shown to result in a transformer ratio $\sim 5$ and peak accelerating field of $E_{+} \sim 60 \mathrm{MV} / \mathrm{m}$ in a dielectric-lined waveguide consistent with the proposal of Ref. [15]. The method offers greater simplicity over other proposed techniques, e.g., based on complex phase-space manipulations $[11,41]$. Finally, we point out that the proposed method could provide bunch shapes consistent with those required to mitigate energy-spread and transverse emittance dilutions due coherent-synchrotron-radiation in magnetic bunch compressors [42].

\section{ACKNOWLEDGMENTS}

We would like to acknowledge members of the ANLLANL-NIU working group on DWFA-based short wavelength FEL led by J. G. Power and A. Zholents for useful discussions that motivated the study presented in this paper. P. P. thanks R. Legg (Jefferson Lab) and J. Bisognano (U. Wisconsin) for providing the $200-\mathrm{MHz}$ quarter-wave field map used in Sec. IV. This work was supported by the U.S. Department of Energy Contract No. DE-SC0011831 to Northern Illinois University, and the Defense Threat Reduction Agency, Basic Research Award \# HDTRA1-101-0051, to Northern Illinois University. P. P. work is also supported by the U.S. Department of Energy under Contract No. DE-AC02-07CH11359 with the Fermi Research Alliance, LLC, and F. L. was partially supported by a dissertation-completion award granted by the Graduate School of Northern Illinois University.

\section{APPENDIX A: MAXIMUM OF $A \cos (k z)+B \sin (k z)$}

The accelerating field behind the bunch often assumes the functional form

$$
F(z)=A \cos (k z)+B \sin (k z) .
$$

The procedure to evaluate the transformer ratio entails determining the maximum value of $F(z)$. Such a value if found by solving for

$$
\frac{d F(z)}{d z}=k[-A \sin (k z)+B \cos (k z)]=0,
$$

with solution $z_{s}$ given by

$$
\tan \left(k z_{s}\right)=\frac{B}{A} \equiv T .
$$

Squaring the previous equation, it is straightforward to show that

$\sin ^{2}\left(k z_{s}\right)=\frac{T^{2}}{1+T^{2}}, \quad$ and $\quad \cos ^{2}\left(k z_{s}\right)=\frac{1}{1+T^{2}}$.

Expressing the value of $F\left(z_{s}\right)$ using the previous equation in (A1) leads to the maximum value of $F(z)$

$$
\hat{F} \equiv F\left(z_{s}\right)=A \sqrt{1+T^{2}} .
$$

The latter equation is used at several instances throughout Sec. II.

\section{APPENDIX B: ANALYTIC DESCRIPTIONS OF THE LINEAR-RAMP AND DOUBLE-TRIANGLE DISTRIBUTIONS}

In this Appendix we summarize and rewrite in notations consistent with our Sec. II the equations describing the linear ramp [6] and double-triangle [10] current profiles. These equations are the ones used in Sec. II C.

The "doorstep" current profile considered in Ref. [6] is written as

$$
S(z)= \begin{cases}a & \text { if } 0 \leq z<\xi, \\ a\left(\frac{2 \pi(z-\xi)}{\lambda}+1\right) & \text { if } \xi \leq z \leq Z, \\ 0 & \text { elsewhere. }\end{cases}
$$

The "double-triangle" suggested in Ref. [10] is given in our notations as

$$
S(z)= \begin{cases}a k z & \text { if } 0 \leq z<\xi, \\ a(k z-1) & \text { if } \xi \leq z \leq Z, \\ 0 & \text { elsewhere }\end{cases}
$$

For both cases, $\xi=\lambda / 4$ leads to the flat decelerating fields over the tail of distribution and leads to the $E_{-}^{m}$ and $\mathcal{R}$ tabulated and illustrated in Table I and Fig. 3, respectively.

[1] P. Chen, J. M. Dawson, R. W. Huff, and T. Katsouleas, Phys. Rev. Lett. 54, 693 (1985).

[2] G. A. Voss and T. Weiland, DESY Hamburg Report No. DESY-M-82-10, 1982.

[3] W. Gai, P. Schoessow, B. Cole, R. Konecny, J. Norem, J. Rosenzweig, and J. Simpson, Phys. Rev. Lett. 61, 2756 (1988).

[4] R. D. Ruth, A. Chao, P. L. Morton, and P. B. Wilson, Part. Accel. 17, 171 (1985). 
[5] V. V. Tsakanov, Nucl. Instrum. Methods Phys. Res., Sect. A 432, 202 (1999).

[6] K. L. F. Bane, P. Chen, and P. B. Wilson, Report No. SLAC-PUB-3662, 1985.

[7] J. G. Power, W. Gai, and P. Schoessow, Phys. Rev. E 60, 6061 (1999).

[8] C. Jing, A. Kanareykin, J. G. Power, M. Conde, Z. Yusof, P. Schoessow, and W. Gai, Phys. Rev. Lett. 98, 144801 (2007).

[9] C. Jing, J. G. Power, M. Conde, W. Liu, Z. Yusof, A. Kanareykin, and W. Gai, Phys. Rev. ST Accel. Beams 14, 021302 (2011).

[10] B. Jiang, C. Jing, P. Schoessow, J. Power, and W. Gai, Phys. Rev. ST Accel. Beams 15, 011301 (2012).

[11] P. Piot, Y.-E Sun, J. G. Power, and M. Rihaoui, Phys. Rev. ST Accel. Beams 14, 022801 (2011).

[12] P. Muggli, V. Yakimenko, M. Babzien, E. Kallos, and K. P. Kusche, Phys. Rev. Lett. 101, 054801 (2008).

[13] Y.-E Sun, P. Piot, A. Johnson, A. H. Lumpkin, T. J. Maxwell, J. Ruan, and R. Thurman-Keup, Phys. Rev. Lett. 105, 234801 (2010).

[14] G. Ha, M. E. Conde, W. Gai, C.-J. Jing, K.-J. Kim, J. G. Power, A. Zholents, M.-H. Cho, W. Namkung, and C.-J. Jing, in Proceedings of the 2014 International Particle Accelerator Conference (IPAC14), Dresden, Germany, 2014, p. 1506.

[15] A. Zholents, W. Gai, R. Limberg, J. G. Power, Y.-E Sun, C. Jing, A. Kanareykin, C. Li, C.X. Tang, D. Yu Shchegolkov, and E. I. Simakov, in Proceedings of the 2014 Free-Electron Laser conference (FEL14), Bassel, Switzerland, 2014, edited by J. Chrin, S. Reiche, and V. Schaa, p. 993.

[16] A. Chao, Physics of Collective Instabilities in High-Energy Accelerators, Wiley Series in Beams \& Accelerator Technologies (John Wiley and Sons, New York, 1993).

[17] F. Lemery and P. Piot, in Proceedings of the 16th Advanced Accelerator Concepts Workshop (AAC 2014), San Jose, CA, 2014 (to be published); also Fermilab Report No. FERMILAB-CONF-14-365-AD, 2014.

[18] G. Andonian, in Proceedings of the 16th Advanced Accelerator Concepts Workshop (AAC 2014), San Jose, CA, 2014 (to be published).

[19] F. Lemery, D. Mihalcea, and P. Piot, in Proceedings of the 3rd International Particle Accelerator Conference, New Orleans, LA, 2012 (IEEE, Piscataway, NJ, 2012), p. 3012.

[20] G. Penco, M. Trovó, and S. M. Lidia, in Proceedings of FEL 2006, BESSY, Berlin, Germany, p. 621.

[21] G. Penco, M. Danailov, A. Demidovich, E. Allaria, G. De Ninno, S. Di Mitri, W. M. Fawley, E. Ferrari, L. Giannessi, and M. Trovó, Phys. Rev. Lett. 112, 044801 (2014).

[22] D. T. Palmer, R. H. Miller, H. Winick, X. J. Wang, K. Batchelor, M. Woodle, and I. Ben-Zvi, in Proceedings of the Particle Accelerator Conference, Dallas, TX, 1995 (IEEE, New York, 1995), p. 982.
[23] K. Flöttmann, AstRA: A Space Charge Algorithm, User's Manual, available from the world wide web at http://www .desy.de/ mpyflo/AstraDokumentation (unpublished).

[24] F. Lemery and P. Piot, in Proceedings of the 2014 International Particle Accelerator Conference (IPAC14), Dresden, Germany, 2014, p. 1454.

[25] O. J. Luiten, S. B. van der Geer, M. J. de Loos, F. B. Kiewiet, and M. J. van der Wiel, Phys. Rev. Lett. 93, 094802 (2004).

[26] A. M. Wiener, Rev. Sci. Instrum. 71, 1929 (2000).

[27] J. W. Gibbs, Nature (London) 59, 606 (1899).

[28] P. Piot, Y.-E Sun, T. J. Maxwell, J. Ruan, E. Secchi, and J. C. T. Thangaraj, Phys. Rev. ST Accel. Beams 16, 010102 (2013).

[29] G. Ferrini, P. Michelato, and F. Parmigiani, Solid State Commun. 106, 21 (1998).

[30] B. Aunes et al., Phys. Rev. ST Accel. Beams 3, 092001 (2000).

[31] R. Legg, W. Graves, T. Grimm, and P. Piot, in Proceedings of the 11th European Particle Accelerator Conference, Genoa, 2008 (EPS-AG, Genoa, Italy, 2008), p. 469.

[32] J. Bisognano, M. Bissen, R. Bosch, M. Efremov, D. Eisert, M. Fisher, M. Green, K. Jacobs, R. Keil, K. Kleman, G. Rogers, M. Severson, D. D. Yavuz, R. Legg, R. Bachimanchi, C. Hovater, T. Plawski, and T. Powers, in Proceedings of the 25th Particle Accelerator Conference, PAC-2013, Pasadena, CA, 2013 (IEEE, New York, 2013), p. 622.

[33] J. Bisognano, R. A. Bosch, D. Eisert, M. V. Fisher, M. A. Green, K. Jacobs, K. J. Kleman, J. Kulpin, and G. C. R. Edit, in Proceedings of the 24th Particle Accelerator Conference, PAC-2011, New York, 2011 (IEEE, New York, 2011), p. 2444.

[34] K. Flöttmann, T. Limberg, and P. Piot, DESY Report No. TESLA FEL 2001-06, 2001.

[35] N. Solyak, I. Gonin, H. Edwards, M. Foley, T. Khabiboulline, D. Mitchell, J. Reid, and L. Simmons, in Proceedings of the 2003 Particle Accelerator Conference, Portland, OR (IEEE, New York, 2003), p. 1213.

[36] M. Borland and H. Shang, GeneticOptimizer (private communication).

[37] I. Bazarov and C. K. Sinclair, Phys. Rev. ST Accel. Beams 8, 034202 (2005).

[38] M. Rosing and W. Gai, Phys. Rev. D 42, 1829 (1990).

[39] P. Emma, Service des Accélérateurs, CEA Saclay, France Report No. DAPNIA/SEA-98-54, 1998.

[40] T. Limberg, Ph. Piot, and F. Stulle, in Proceedings of the 8th European Particle Accelerator Conference, Paris, 2002 (EPS-IGA and CERN, Geneva, 2002), p. 1544.

[41] P. Piot, C. Behrens, C. Gerth, M. Dohlus, F. Lemery, D. Mihalcea, P. Stoltz, and M. Vogt, Phys. Rev. Lett. 108, 034801 (2012).

[42] C. Mitchell, J. Qiang, and P. Emma, Phys. Rev. ST Accel. Beams 16, 060703 (2013). 